\title{
Hypermotor seizures without involvement of the frontal lobes
}

\author{
Sikawat Thanaviratananich ${ }^{\mathrm{a}, *}$, Asim Shahid $^{\mathrm{b}}$, Hans O. Lüders ${ }^{\mathrm{a}}$ \\ a Comprehensive Epilepsy Center Neurological Institute, University Hospitals Cleveland Medical Center, Case Western Reserve University, Cleveland, OH, 44106, \\ USA \\ bivision of Pediatric Epilepsy, University Hospitals Cleveland Medical Center, Rainbow Babies and Children's Hospital, Cleveland, OH, 44106, USA
}

A R T I C L E I N F O

\section{Article history:}

Received 29 May 2017

Accepted 10 August 2017

Available online 16 August 2017

\section{Keywords:}

Hyperkinetic seizure

Symptomatogenic zone

Frontal lobe epilepsy

\section{Introduction}

Hypermotor seizures or hyperkinetic seizures according to the ILAE seizure glossary ${ }^{1}$ are characterized by automatisms affecting mainly the proximal muscles and resulting in relatively "violent" movements. ${ }^{1,2}$ Hypermotor seizures consist of pelvic thrashing, body rocking, bicycling, pedaling, kicking, or violent clapping. Even though there is no conclusive proof on the location of the symptomatogenic zone of hypermotor seizures, it has generally been assumed to be in the frontal lobe, mostly the anterior cingulate gyrus ${ }^{3,4}$ or the orbitofrontal cortex ${ }^{5}$, or both. ${ }^{6}$

Here we report two cases of hypermotor seizures of temporal lobe and insula origin. Both cases had extensive coverage of the frontal lobes with depth electrodes and in both cases the patient experienced hypermotor symptoms without any evidence of seizure onset in the frontal lobe or spread of the epileptiform discharge to frontal lobe depth electrodes at the time he experienced hypermotor symptoms. This makes it unlikely that in these patients the symptomatogenic zone for hypermotor symptomatology was localized in the frontal lobe.

\section{Case study}

\subsection{Case 1}

A 39-year-old man presented with refractory nocturnal paroxysmal episodes since age 13 . His wife reported that he suddenly awoke from sleep exhibiting violent movements such as

\footnotetext{
* Corresponding author.

E-mail address: thanavir@gmail.com (S. Thanaviratananich).
}

punching the pillow accompanied by vocalization during some of these episodes. Each episode lasted for approximately $1 \mathrm{~min}$ and he had no recollection of these episodes. He had been treated unsuccessfully with levetiracetam and oxcarbazepine. MRI of the brain with an epilepsy protocol was unremarkable.

His first admission in our EMU with monitoring of noninvasive EEG with additional sphenoidal electrodes (Sp) revealed interictal sharp waves with maximum negativity at the right sphenoidal electrode (Sp2). Two of his typical episodes were recorded. With these seizures while asleep he suddenly had violent proximal complex movements followed by oral automatisms. This lasted for $25 \mathrm{~s}$ and evolved into a left versive seizure followed by a secondarily generalized tonic-clonic seizure lasting for approximately $1 \mathrm{~min}$. There was postictal confusion lasting for $30 \mathrm{~min}$. The ictal EEG revealed seizures arising from Sp2.

Five months later he underwent stereotactic depth electrodes implantation. Nine depth electrode contacts with a diameter of $1.3 \mathrm{~mm}$, a contact length of $2.5 \mathrm{~mm}$, and a centre-to-centre distance of $5.0 \mathrm{~mm}$, were stereotactically placed in the right hemisphere including the inferior frontal lobe (IF), the temporal pole (TP), the amygdala (AM), the hippocampal body (HB), the hippocampal head $(\mathrm{HH})$, the anterior insula (AI), the posterior insula (PI), the posterior temporal (PT) region and the cingulate gyrus $(\mathrm{CI})$. Five additional frontal electrodes (designated FA, FB, FC, FD and FE from rostral to caudal in the frontal lobe and IF for an inferior frontal electrode) and one additional anterior insular (FF) electrode were later implanted on the right hemisphere and electrodes CI and PT were removed at the same time (Fig. 1). The trajectory and placement of the electrodes were demonstrated using postoperative CT scan of the head coregistered with a presurgical volumetric preoperative MRI by iPlan 2.6 Cranial software (BrainLAB). 


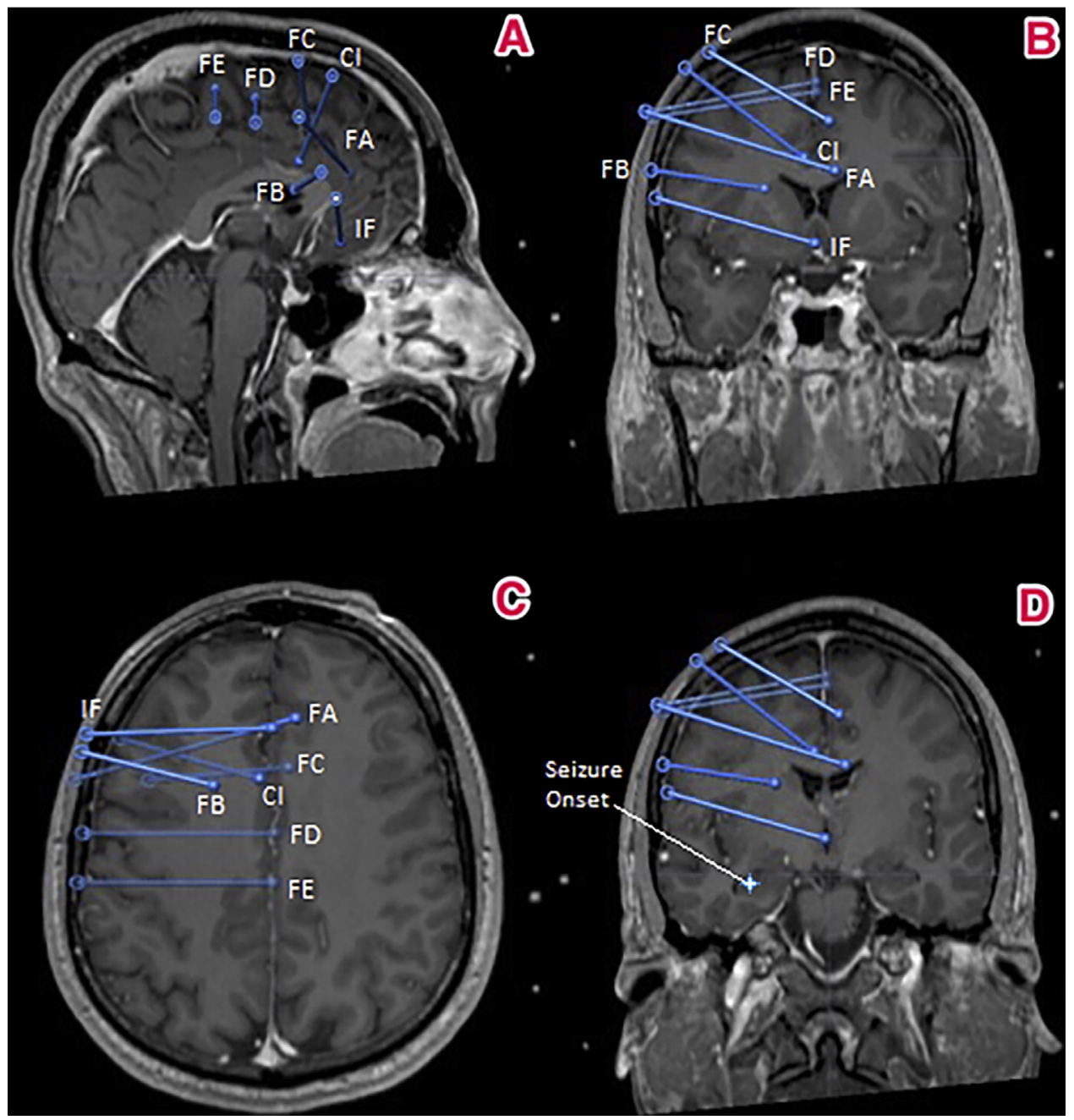

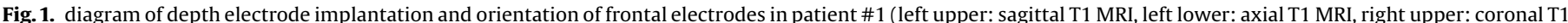

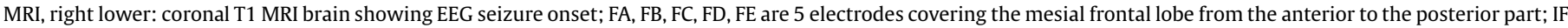
is an inferior frontal lobe electrode, and $\mathrm{CI}$ is a cingulate electrode).

Interictal spikes were localized to the right $\mathrm{HH}$ (HH1-4; one spike every minute), right $\mathrm{HB}$ (HB1-3; one spike every $3 \mathrm{~min}$ ) and the right amygdala (AM1-4; one spike every $10 \mathrm{~min}$ ). Three epileptic seizures were recorded. During all 3 seizures, the patient awoke from sleep and started punching the pillow or the bed with unintelligible loud vocalization. This was followed by a period of lip smacking. During the first seizure, the patient was unresponsive through the lip smacking, but later was able to answer questions appropriately even while the electrographic seizure was still going on. In the second seizure the patient did not respond verbally until after the seizure activity stopped. There was no postictal confusion. The clinical onset preceded the EEG seizure onset consistently by 1-3 s. The semiological classification was hypermotor seizure (with loss of awareness) - > automotor seizure. The ictal onset was at the right hippocampal head (HH3) and right hippocampal body (HB2) (Figs. 2 and 3 ). Figs. 2 and 3 showed one of his seizures. The EEG seizure onset was located at HH3 and HB2. During this event, the clinical seizure started by the patient waking up $1 \mathrm{~s}$ before the EEG seizure onset. Then he developed whole body shaking and started punching the pillow (hypermotor seizure). At the time of the clinical hypermotor seizure, the seizure discharge did not involve any of the frontal electrodes despite the extensive coverage (FA, FB, FC, FD, FE, and IF) of the frontal lobe with depth electrodes.
The clinical onset preceded the EEG seizure onset implying that the ictal onset zone was located in an area not being covered by the depth electrodes. A right temporal lobectomy was performed and the pathology revealed focal cortical dysplasia, Palmini grade $1 \mathrm{~A}$. The patient was discharged on levetiracetram and oxcarbazepine. On a 5-year follow-up, he reported having a single nocturnal "tremulous episode" 5 months ago which was uncertain if it was an epileptic seizure. He denied having any generalized tonic-clonic seizure since the surgery. The epileptogenic zone was likely in the resected area, but not in the hippocampus in this case.

\subsection{Case 2}

A 15-year-old woman, with seizures since age 8, was admitted to our Pediatric EMU. Her seizures started with a feeling of vibration and tingling in the left hemibody, followed by facial grimacing, laughing, limbs flailing, and side-to-side movements of the trunk with preserved awareness during the whole episode, lasting up to a minute without postictal confusion. The seizures were refractory to valproic acid and levetiracetam. A high resolution brain MRI with thin cuts through the sella was obtained due to the gelastic seizure component. This MRI was unrevealing ruling out a hypothalamic hamartoma. During monitoring, her EEG 


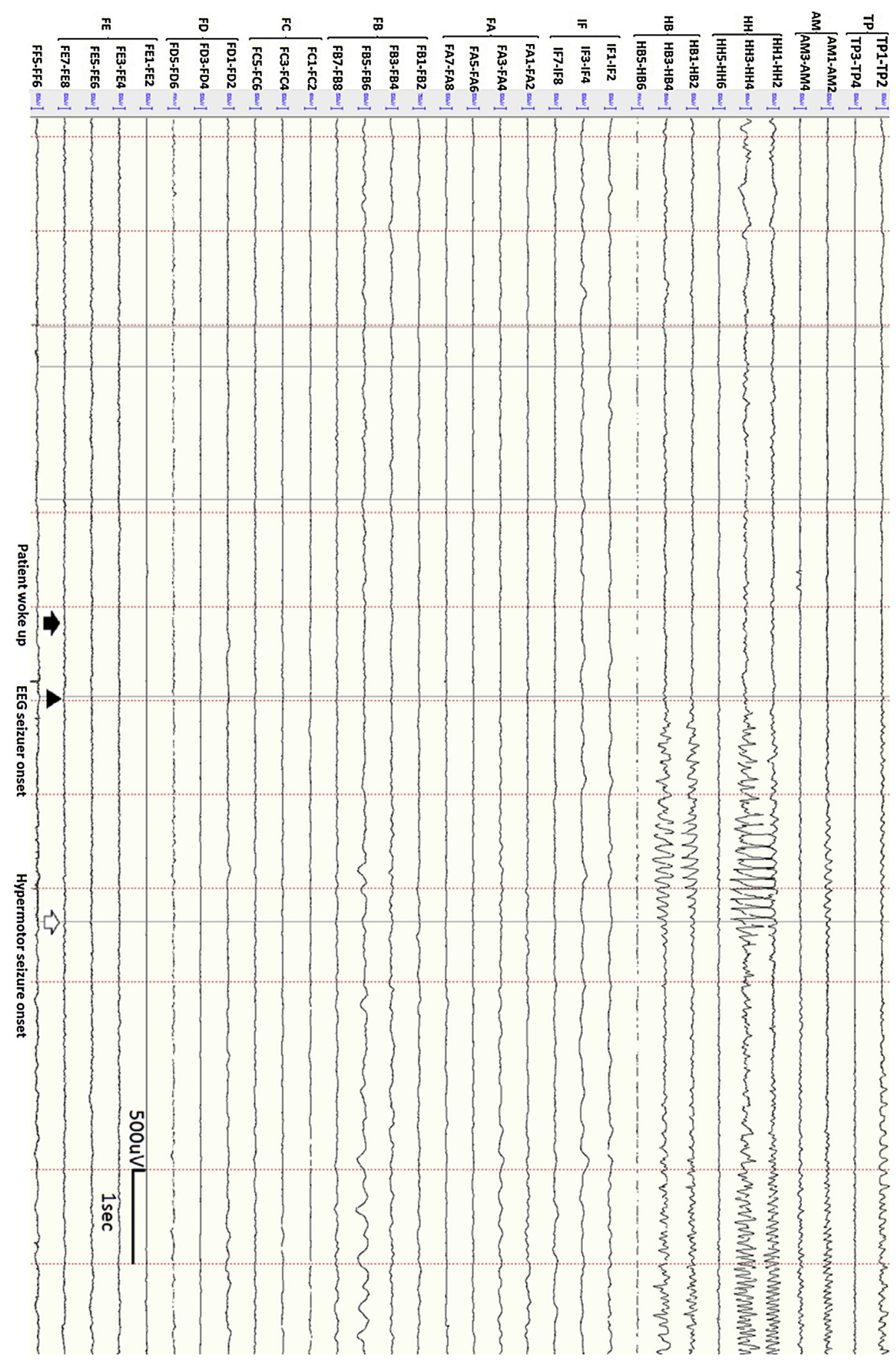

Figs. 2 and 3. Invasive EEG monitoring of patient\#1 showing the typical evolution of a clinical seizure. The EEG seizure started and evolved involving almost exclusively the mesial and anterior temporal electrodes (AM, HH, HB and TP). The patient's clinical seizure started with the patient waking up (black arrow in Figure2 Fig. 2) $1 \mathrm{~s}$ before the EEG seizure onset (arrowhead Then when the patient developed whole body shaking (empty arrow and started punching the pillow accompanied by vocalization (black arrow in Fig. 3) there was no involvement of any frontal electrodes, despite extensive coverage of the frontal lobe. Of note, some of the channels are not shown due to inactivity even after the gain was increased. (See Fig. 1 for the nomenclature of the electrodes).

showed interictal spikes in the right frontal region and intermittent slow in the right fronto-central region. In addition, the background activity was decreased in amplitude in the right fronto-central region $(20-30 \mu \mathrm{V}$ over the right fronto-central region and $50-60 \mu \mathrm{V}$ over the left fronto-central region). The seizures started with vibration and tingling in the left hemibody, 

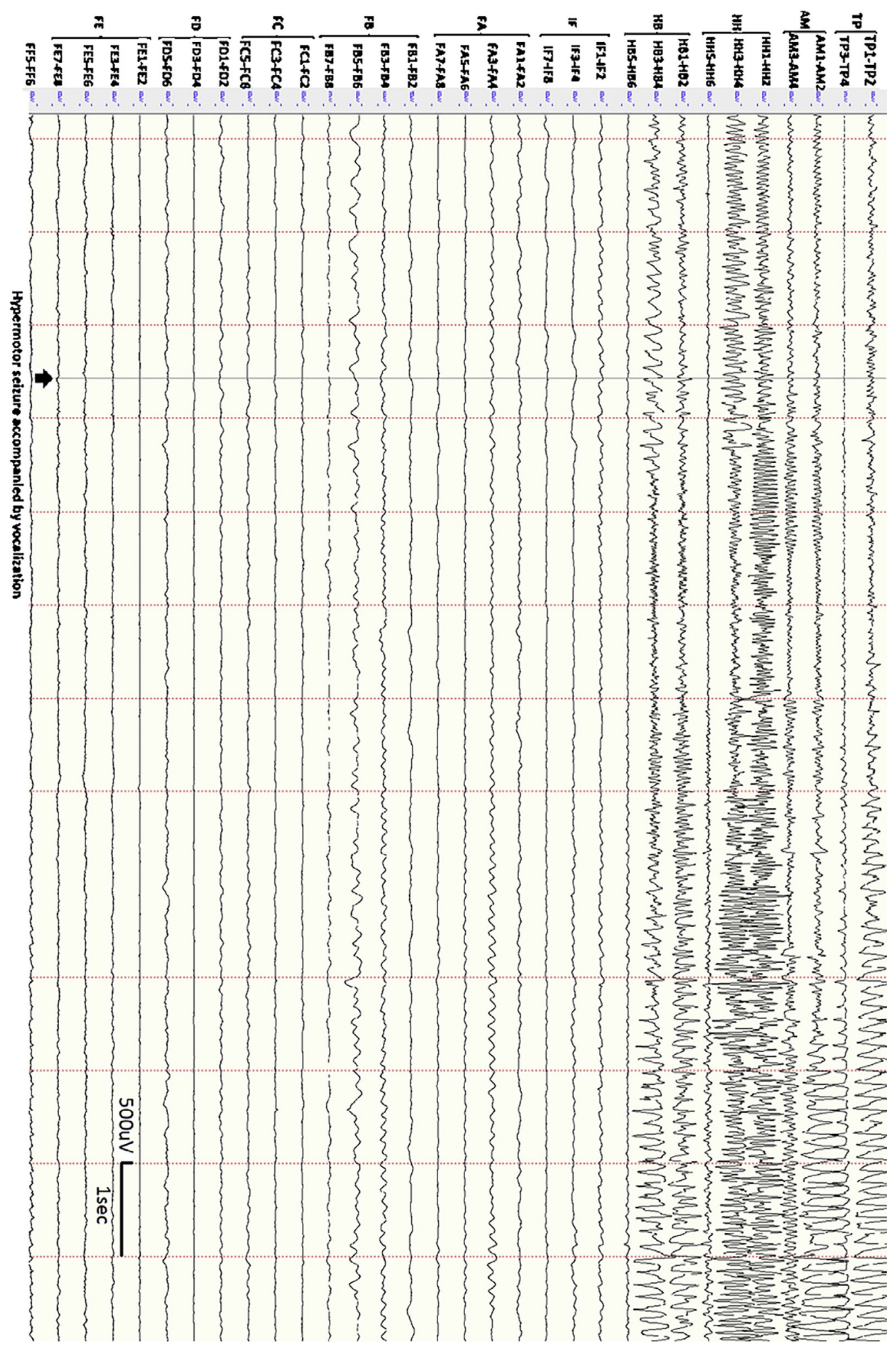

Figs. 2 and 3. (Continued)

followed by bicycling movements (hypermotor seizure). Thirty seizures were recorded. The ictal EEG revealed the seizures emanating from the midline and right fronto-central regions. After multiple medication failures, she was discussed in our patient management conference with a plan to have extensive frontal lobe depth electrode implantation, sampling the premotor frontal lobe in a $3 \times 3$ grid from the parasagittal to the lateral cortex (just above the Sylvian fissure) with 2 additional electrodes in the anterior and the posterior insula (AI and PI respectively) (Fig. 4). The insular electrodes were implanted due to the tingling and vibratory aura. 


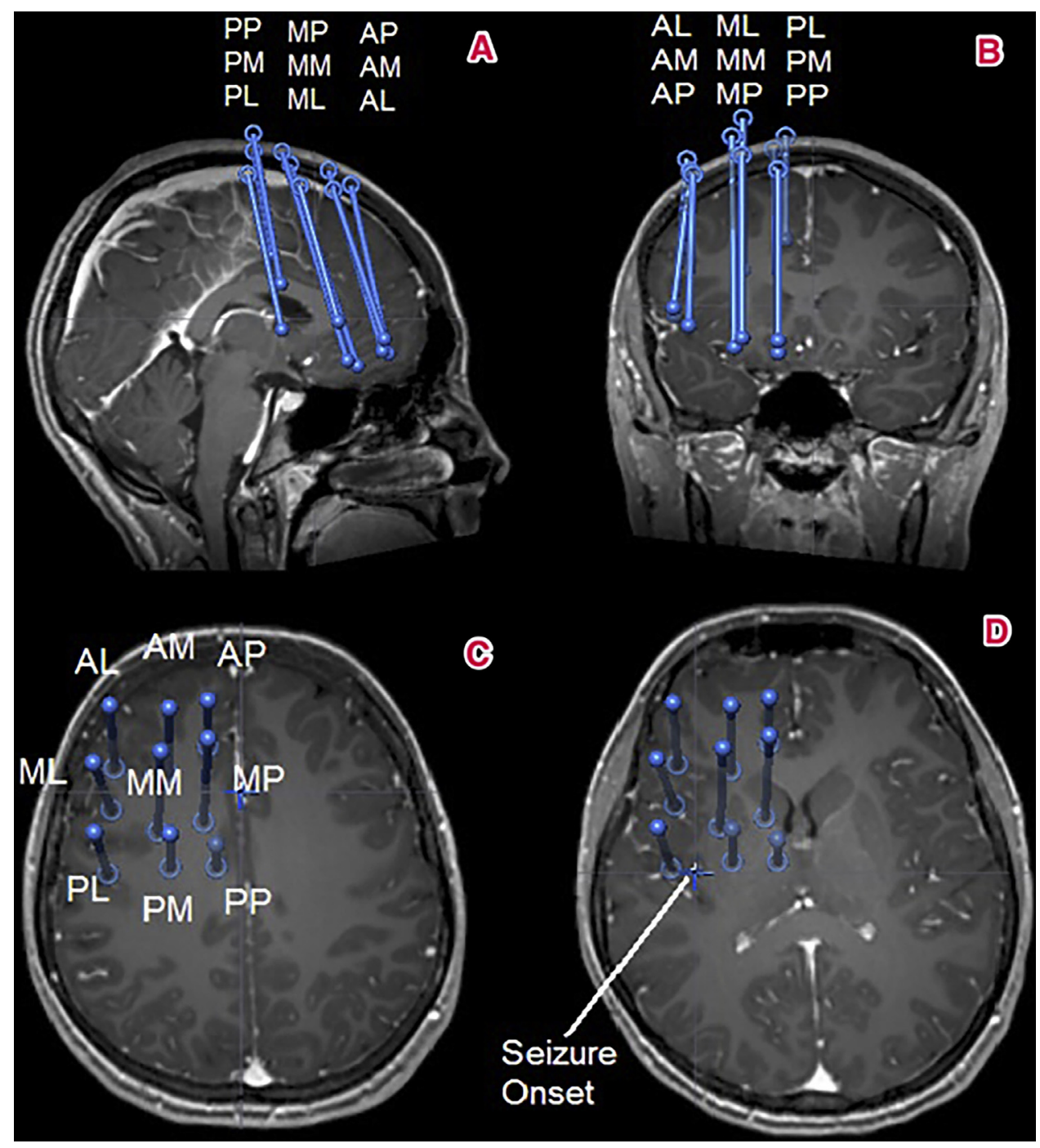

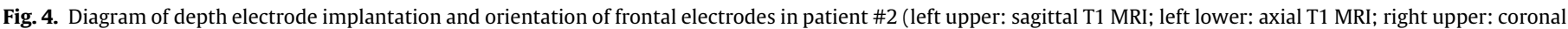

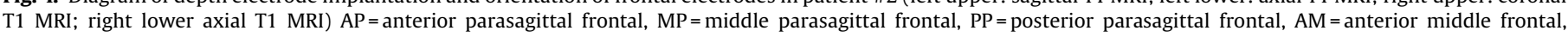
$\mathrm{MM}=$ middle middle frontal, $\mathrm{PM}=$ posterior middle frontal, $\mathrm{AL}=$ anterior lateral frontal, $\mathrm{ML}=$ middle lateral frontal, $\mathrm{PL}=$ posterior middle frontal).

Each electrode had 12 contacts. The trajectory and placement of the electrodes were planned and placed with the same technique as in case \#1. Interictal discharges were seen in electrodes PI3-8 and posterior parasagittal (PP) electrodes 5-12. Seizure onset was seen in a very small region of the posterior superior aspect of the insula (PI3-8) (Fig. 5). There was no spread of the ictal activity to any of the 9 frontal lobe electrodes even during the hypermotor phase of the seizure. Electrodes were explanted. No surgery or additional depth electrode studies were done according to the family's request.

\section{Discussion}

The symptomatogenic zone is defined as the cortical area which when activated by an epileptic seizure produces the ictal symptomatology. For hypermotor seizure, it is generally assumed to be located in the frontal lobe, mostly the anterior cingulate gyrus $^{3,4}$, or the orbitofrontal cortex. ${ }^{5}$ Several authors reported that in patients with temporal lobe epilepsy, the hypermotor symptomatology appeared once the discharge propagated from the temporal to the cingulate gyrus, mesial frontal, orbitofrontal or parietal lobe. ${ }^{7,8}$ Some authors also noticed that in patients with insula epilepsy and hypermotor seizures, the hypermotor behavior occurred when the discharge spread from the insula to involve the mesial frontal lobe or cingulate gyrus. ${ }^{9,10}$ Based on these reports, investigators assumed that the symptomatogenic zone for hypermotor seizures was located in the frontal lobe and/or anterior cingulate gyrus. On the other hand, other authors suggested that the complex motor movements seen during hypermotor seizures are not specifically associated with the activation of any subregion of the frontal lobe ${ }^{11,12}$ but may be the expression of a disruption of the inhibition of higher brain centers over subcortical motor centers leading to primitive behaviors. ${ }^{13}$

It is important to remember, however, that very frequently ictal discharges may involve eloquent cortical regions without producing any signs or symptoms. Therefore, the fact that an epileptic discharge reaches a certain cortical region simultaneously with the appearance of a given symptomatology certainly does not mean that cortical region is the symptomatogenic zone for that ictal symptom or sign. In other words, there is a large margin of error 


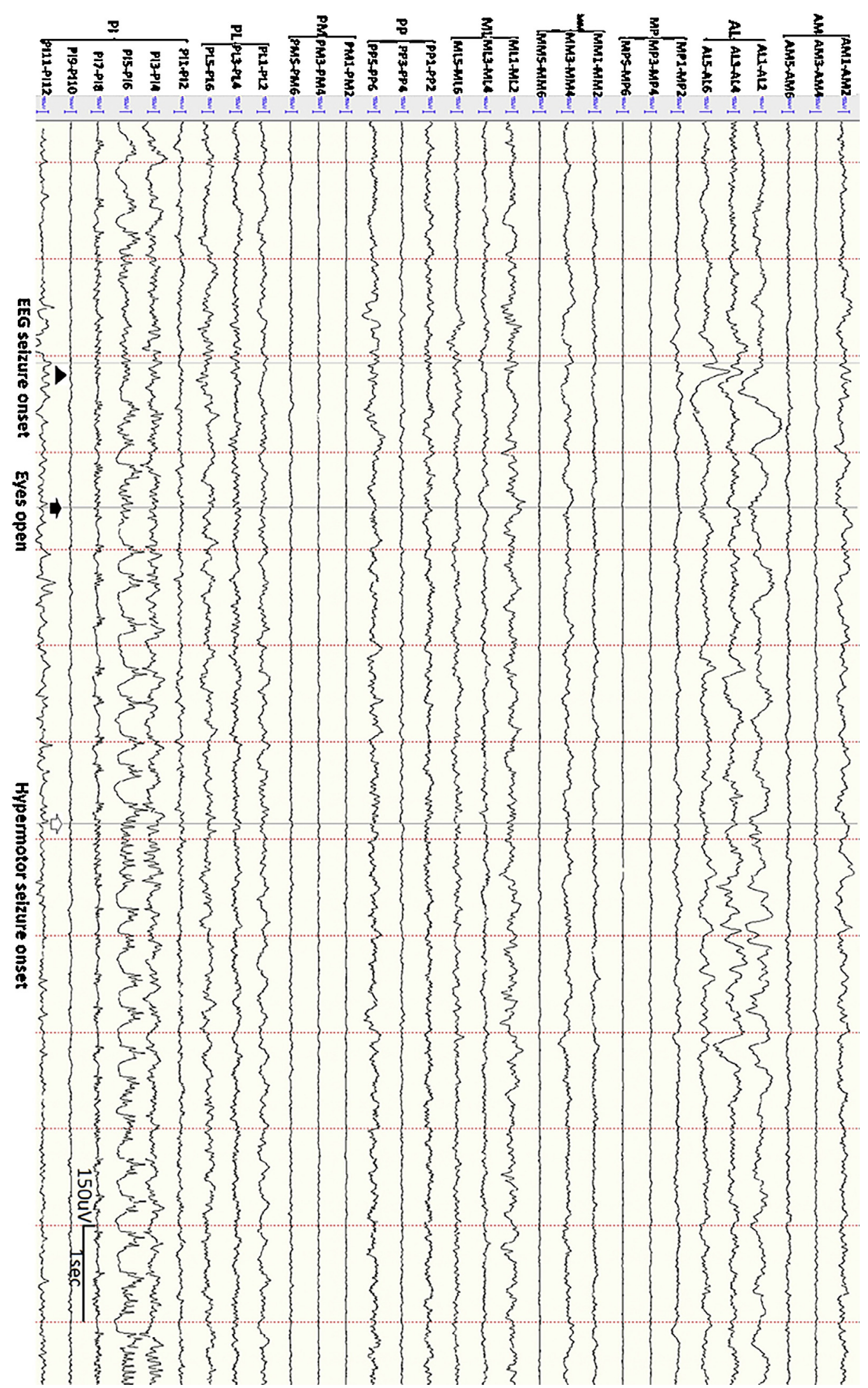

Fig. 5. Invasive EEG monitoring in patient \#2 showing seizure onset zone in the right posterior insular electrodes (PI3-8). EEG seizure onset was marked by an (arrowhead) The patient opened her eyes from sleep (black arrow) $1 \mathrm{~s}$ after EEG onset and then developed non-purposeful, large amplitude legs and arms movements (empty arrow) At the time of the hypermotor seizures and actually throughout the clinical seizure the EEG seizure activity was confined to the posterior insular electrodes with no involvement of any frontal electrodes. (See Fig. 4 for the nomenclature of the electrodes). 
when trying to elucidate the symptomatogenic zone by studying the sequence of ictal symptomatology and its temporal relationship to the spreading of the epileptiform discharge.

On the other hand, we can exclude with confidence that a specific cortical region is the symptomatogenic zone for a given ictal symptom if during a seizure we do not detect any epileptiform discharge involving that specific cortical region. In the 2 cases described above, none of the frontal depth electrodes revealed any epileptiform discharges at the time the patients experienced hypermotor symptomatology. This strongly suggest, that at least in some cases of temporal or insular lobe epilepsy, no spread of the epileptiform discharge into the frontal or cingulate lobe is necessary to elicit hypermotor symptomatology.

This study has some limitations. In both our cases, there was only limited coverage of the cingulate gyrus. Alkawadri et al. recently reported a case with mid-cingulate epilepsy in which the electrographic seizure was limited to only the mid-cingulate electrode contacts without involvement of other frontal lobe electrodes while the patient was experiencing hypermotor symptomatology. ${ }^{14}$ However, in both our cases, the seizures came from an extrafrontal lobe. Therefore, it is very unlikely that the epileptiform discharges would selectively spread to the cingulate gyrus without showing any involvements in any of the adjacent electrodes covering the frontal lobe. Besides, considering that the frontal lobes consist of almost $50 \%$ of the brain, it is practically impossible to cover all frontal cortexes with depth electrodes. However, in these two cases we had a relatively extensive coverage of the frontal lobe with depth electrodes and no epileptiform discharges were seen in frontal depth electrodes when the patients experienced hypermotor symptoms. This makes it very unlikely that in these cases the frontal cortex was the symptomatogenic zone for the hypermotor symptomatology.

This study shows that most likely activation of the frontal cortex is not necessary for generation of hypermotor symptomatology in certain patients. The study does not specify the pathophysiology of hypermotor symptomatology. It is interesting to observe, however, that cortical stimulation only generates hypermotor symptomatology when the stimulation elicits afterdischarges or clear epileptic seizures. This observation supports Williamson and Jobst's conclusion ${ }^{11,12}$ that hypermotor seizures may not be the expression of an activation of a specific brain region but a release phenomenon of subcortical motor centers from their cortical control.

Our case reports strongly suggest that the symptomatogenic zone of the hypermotor seizures our patients had experienced was located outside the frontal lobe. This observation, however, does not necessarily implies that the frontal lobe may be the symptomatogenic zone for hypermotor seizures in other patients.

\section{Ethical publication statement}

We confirm that we have read the Journal's position on issues involved in ethical publication and affirm that this report is consistent with those guidelines.

\section{Conflict of interest}

None.

\section{Acknowledgement}

None and all authors have no disclosure.

\section{References}

1. Blume WT, Lüders HO, Mizrahi E, Tassinari C, van Emde Boas W, Engel J. Glossary of descriptive terminology for ictal semiology: report of the ILAE task force on classification and terminology. Epilepsia. 2001;42(September (9)):1212-1218.

2. Lüders H, Acharya J, Baumgartner C, et al. Semiological seizure classification. Epilepsia. 1998;39(September (9)):1006-1013.

3. Tao Y, Guojun Z, Yuping W, Lixin C, Wei D, Yongjie L. Surgical treatment of patients with drug-resistant hypermotor seizures. Epilepsia. 2010;51(October (10)):2124-2130.

4. San Pedro EC, Mountz JM, Ojha B, Khan AA, Liu HG, Kuzniecky RI. Anterior cingulate gyrus epilepsy: the role of ictal rCBF SPECT in seizure localization. Epilepsia. 2000;41(May (5)):594-600.

5. Bartolomeil F, Guye M, Wendling F, Gavaret M, Régis J, Chauvel P. Fear, anger and compulsive behavior during seizure: involvement of large scale frontotemporal neural networks. Epileptic Disord Int Epilepsy J Videotape. 2002;4 (December (4)):235-241.

6. Rheims S, Ryvlin P, Scherer C, et al. Analysis of clinical patterns and underlying epileptogenic zones of hypermotor seizures. Epilepsia. 2008;49(December (12)):2030-2040.

7. Nobili L, Cossu M, Mai R, et al. Sleep-related hyperkinetic seizures of temporal lobe origin. Neurology. 2004;62(February (3)):482-485.

8. Wang L, Mathews GC, Whetsell WO, Abou-Khalil B. Hypermotor seizures in patients with temporal pole lesions. Epilepsy Res. 2008;82(November (1)):9398.

9. Ryvlin P, Minotti L, Demarquay G, et al. Nocturnal hypermotor seizures suggesting frontal lobe epilepsy, can originate in the insula. Epilepsia. 2006;47 (April (4)):7553-7765.

10. Proserpio P, Cossu M, Francione S, et al. Insular-opercular seizures manifesting with sleep-related paroxysmal motor behaviors: a stereo-EEG study. Epilepsia. 2011;52(October (10)):1781-1791.

11. Williamson PD, Jobst BC. Frontal lobe epilepsy. Adv Neurol. 2000;84:215-242.

12. Jobst BC, Siegel AM, Thadani VM, Roberts DW, Rhodes HC, Williamson PD. Intractable seizures of frontal lobe origin: clinical characteristics, localizing signs, and results of surgery. Epilepsia. 2000;41(September (9)):1139-1152.

13. McGoniaGal A, Chauvel P. Frontal lobe epilepsy: seizure semiology and presurgical evaluation. Pract Neurol. 2004;10:260-273.

14. Alkawadri R, Gonzalez-Martinez J, Gaspard N, Alexopoulos AV. Propagation of seizures in a case of lesional mid-cingulate gyrus epilepsy studied by stereoEEG. Epileptic Disord Int Epilepsy J Videotape. 2016;18(December (4)):418-425. 